\title{
Deformationsmechanische Kennwerte von Kunststoffen für die Festigkeits-, Formänderungs- und Stabilitätsrechnung*
}

\author{
Bernd-Rüdiger Meyer
}

\section{Aufgabenstellung}

Mechanisch beanspruchte Kunststoffbauteile müssen ausreichend funktionssicher sowie wirtschaftlich gestaltet und dimensioniert werden, um den ständig steigenden Marktanforderungen zu genügen. Bereits im Entwurfsstadium beginnt ein Optimierungsprozeß, der je nach Randbedingungen (Werkstoffpreis, Sicherheitsbedürfnis, Erzeugnismenge u. a.) mit einer mehr oder weniger aufwendigen Erzeugnisprïfung bzw. Erprobung abschließt. Für hochfeste Faser-Kunststoff-Verbundkonstruktionen und ähnlich teure Konstruktionsteile ist diese Verfahrensweise selbstverständlich. Hingegen sind bei vielen Kunststoffanwendungen geringerer Wertigkeit mit relativ wenig Aufwand noch erhebliche Reserven zu erschließen.

Kunststoffe, wie auch andere Polymerwerkstoffe, gehören zur Klasse der viskoelastischen Werkstoffe, deren Deformations- und Werkstoffschädigungsverhalten erheblich komplexer als das der reinelastischen oder elastisch-plastischen Werkstoffe (z. B. Metalle) ist. Allgemein lassen sich viskoelastische Stoffe durch die gleichzeitige Wirkung energieelastischer, entropieelastischer und viskoser Verformungsmechanismen charakterisieren, die je nach Art der Lasterregung (Retardation, Relaxation) u. U. unterschiedlich reagieren. Eine Viskoelastizitätstheorie in geschlossener Form liegt nur für den linearviskoelastischen Bereich vor /1/. Aber bereits diese Theorie erfordert selbst bei Vereinfachungen relativ aufwendige mathematische Formalismen und Datenstrukturen, ist aber im üblichen Verfomungsbereich von Duroplastwerkstoffen anwendbar. Bei voller Ausnutzung der mechanischen Belastbarkeit vieler Thermoplaste kommt man zunehmend in den nichtlinearviskoelastischen Bereich, für den die theoretische Mechanik nur Näherungshypothesen zur Verfuigung stellt /1/.

Wenn die praktische Anwendung der Viskoelastizitätstheorie beurteilt werden soll, muß man sich vergegenwärtigen, daß bis zum heutigen Tag die meisten Entwickler und Konstrukteure von Kunststoffteilen während ihrer ingenieurtechnischen Ausbildung nur die technische Mechanik des Hookschen Gesetzes und die Statik am unverformten Bauteil kennengelernt haben. Weiterhin war in der Anfangszeit der Kunststoffentwicklung der mathematische Aufwand bei der praktischen Anwendung der Viskoelastizitätstheorie ein Hindernis. Dieses

\footnotetext{
- Vortrag zur TECHNOMER '97 - 15. Fachtagung über Verarbeitung und Anwendung von Polymeren, 13.-15.11.1997, Technische Universität Chemnitz
}

Problem wäre zwar heute durch die Computertechnik lösbar, sofern die erforderlichen Stoffdaten verfügbar sind. Eine hinreichend vollständige Prüfung des zeit- und temperaturabhängigen Verformungs- und Werkstoffschädigungsverhaltens der Kunststoffe ist bei deren großer Typenvielfalt mit wirtschaftlich vertretbarem Aufwand nur fuir Ausnahmefälle möglich.

Die vorgenannnten Gründe sind u. a. dafür maßgebend, daß die praktische Berechnung von Kunststoffteilen überwiegend mit den üblichen Lösungen der Elastizitätslehre erfolgt, in denen der Hooksche Elastizitätsmodul durch den zeit-, temperatur-, spannungs- bzw. dehnungsabhängigen Kriech- oder Relaxationsmodul ersetzt wird. Diese Vorgehensweise soll hier als Prinzip der elastischen Näherung bezeichnet werden. Gültigkeitsbereich und Kennwerte dieser einfachen und anschaulichen Methode sind Gegenstand der folgenden Ausführungen. Dabei wird nur auf die Spannungs-Verformungs-Zuordnung im weitgehend schädigungsfreien Bereich der Werkstoffdeformation eingegangen. Weiterhin beschränken sich die Ausführungen auf isotrope und homogene Werkstoffe, deren stoffliche Struktur sich durch chemische oder physikalische Alterungseinflüsse während der Beanspruchungszeit nicht wesentlich ändert.

\section{Deformationskennwerte und Gültigkeits- bereich der elastischen Näherung}

Für die folgenden Betrachtungen werden isochrone Spannungs-Dehnungs-Diagramme (ISD) aus dem einachsigen statischen Zugkriech- oder Zugrelaxationsversuch (Bild 1) bei unterschiedlichen Temperaturen vorausge-

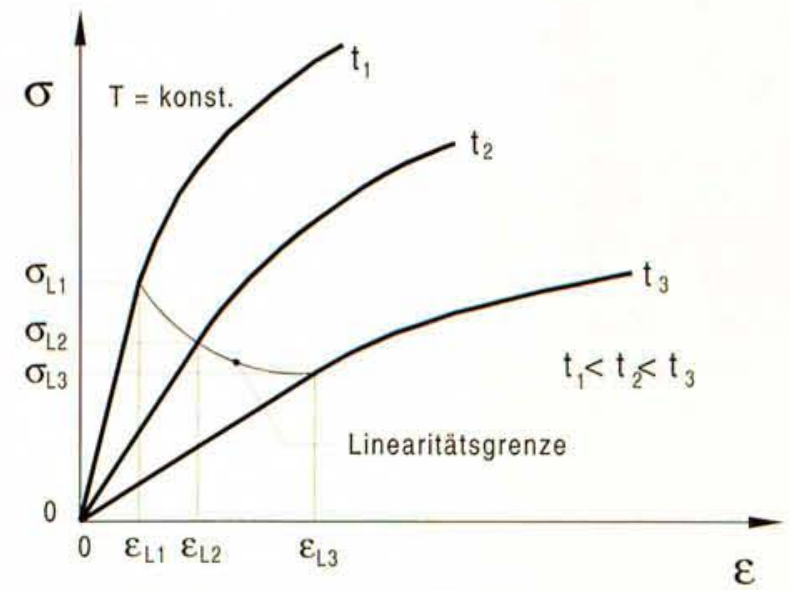

Bild 1: Isochrones $\sigma-\varepsilon-D i a g r a m m$ (schematisch) 
setzt, die der Kunststoffanwender aus Datenbanken, Firmenschriften der Kunststoffhersteller u. a. entnehmen kann.

Auf Grund des höheren Prüfaufwandes werden Relaxationsversuche vergleichsweise selten durchgeführt, so daß der Nutzer häufig nur Kriechkennwerte zur Verfügung hat. Die ISD-Verläufe können durch verschiedene Anstiegsmoduli beschrieben werden, deren geometrische Bedeutung aus Bild 2 zu entnehmen ist.

Für zeitabhängige Modulwerte gilt dann die Relation $\mathrm{E}_{0(\mathrm{t})} \geq \mathrm{E}_{\mathrm{S}(\mathrm{t})} \geq \mathrm{E}_{\mathrm{T}(\mathrm{t})}$ bei jeweils konstanter Temperatur: Der Sekantenmodul aus dem Kriech- bzw. Retardationsversuch ( $\sigma$ vorgegeben) wird mit $\mathrm{E}_{\mathrm{c}(1)}=\frac{\sigma}{\varepsilon_{(1)}}$ als Kriechmodul und der aus dem Relaxationsversuch ( $\varepsilon$ vorgegeben) mit $E_{r(1)}=\frac{\sigma_{(1)}}{\varepsilon}$ als Relaxationsmod bezeichnet. Im linearviskoelastischen Bereich gehen die Modulwerte in den Ursprungsmodul $\mathrm{E}_{0(\mathrm{t})}$ über:

$$
\begin{aligned}
& \lim \mathrm{E}_{S} ; \mathrm{T}(\mathrm{t})=\mathrm{E}_{0(\mathrm{t})} \\
& \varepsilon \leq \varepsilon_{\mathrm{L}(\mathrm{t})} ; \sigma \leq \sigma_{\mathrm{L}(\mathrm{t})}
\end{aligned}
$$

Dabei sind Linearitätsdehnung $\varepsilon_{\mathrm{L}}$ bzw. Linearitätsspannung $\sigma_{\mathrm{L}}$ ebenfalls zeit- und temperaturabhängige Kennwerte.

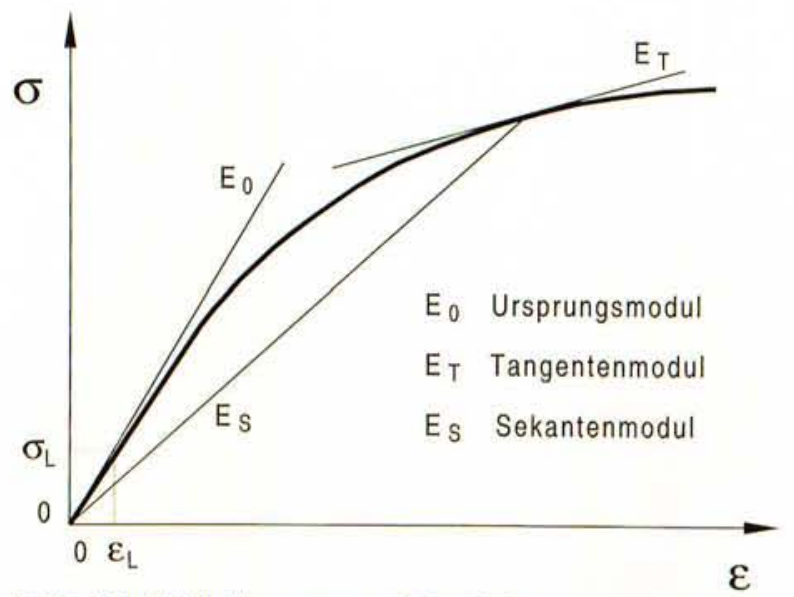

Bild 2: E-Moduldefinition aus geometrischer Sicht

\subsection{Einachsige Membranspannungsbeanspruchung}

Werden Bauteilquerschnitte durch konstante Zug- oder Druckspannungen (Membranspannungen) belastet, so läßt sich die elastische Näherung der Spannungs-Verformungs-Zuordnung wie folgt formulieren:

$$
\sigma=\mathrm{E}_{\mathrm{c} ; \mathrm{r}(\mathrm{t})} \cdot \varepsilon
$$

Bei Anwendung von Glg. (1) sind aber Voraussetzungen zu beachten, die deren Gültigkeit unter Umständen erheblich einschränken können. Zur besseren Veranschaulichung dieser Zusammenhänge ist folgende Schreibweise vorzuziehen:

$$
\sigma=\mathrm{E}_{0(\mathrm{t})} \cdot \mathrm{L} \cdot \varepsilon
$$

In Glg. (2) soll L als Linearitätsfaktor $(0<\mathrm{L} \leq 1)$ definiert werden. Dieser Faktor quantifiziert anschaulich die Linearitätsabweichung der $\sigma$ - $\varepsilon$-Diagramme. Bei Anwendung der Glg. (2) müssen bereits im linear-viskoelastischen Bereich
( $\mathrm{L}=1$ ) Gültigkeitsvoraussetzungen beachtet werden, die aus den physikalischen Grundtatsachen der Viskoelastizitätstheorie ableitbar sind /1/.

1. Die Ursprungsmoduli für Kriech- und Relaxationsbelastung müssen zahlenmäßig annähernd gleich sein. Wie die Auswertung experimenteller Untersuchungen durchweg ergibt, kann diese Forderung für alle halbharten bis harten Kunststoffe $\left(\mathrm{E}_{0(\mathrm{t})}>300 \mathrm{~N} / \mathrm{mm}^{2}\right)$ als erfuillt gelten. Die Abweichungen betragen meist deutlich weniger als $10 \%$.

$$
\mathrm{E}_{0 \mathrm{c}(\mathrm{t})} \approx \mathrm{E}_{0 \mathrm{r}(\mathrm{t})}=\mathrm{E}_{0(\mathrm{t})}
$$

Für ausgeprägt gummielastische oder weichelastische Polymere ist diese Forderung a priori nicht immer erfuillt.

2. Die Belastungsgröße $(\sigma ; \varepsilon)$ darf sich nach ihrer Aufbringung zeitlich nicht (statische Belastung) oder nur so geringfuigig ändern, daß sie sich zur Molekülrelaxation immer in einem angenäherten Gleichgewichtszustand befindet (quasistatische Belastung). Seltene Lastspitzen bei insgesamt langer Gesamtbelastungszeit können meist vernachlässigt werden. Bei sich wiederholenden periodischen Lastzyklen (z. B. periodisch intermittierende Belastung) stellt sich nach genügend großen Lastwechselzahlen ein quasistatisches Gleichgewicht ein. Auf die Besonderheiten dynamischer Beanspruchung, z. B. schwingende Beanspruchung bei Lastfrequenzen $f>1$ $\mathrm{Hz}$, wird im Rahmen dieses Vortrages nicht eingegangen. Der zügige Standardzugversuch mit variabler Dehngeschwindigkeit (zügiger Relaxationsversuch) erfüllt nicht die Bedingungen der elastischen Näherung.

3. Im nichtlinearviskoelastischen Bereich $(\mathrm{L}<1)$ sind aus der Viskoelastizitätstheorie nur Vermutungen zur Gültigkeit der elastischen Näherung abzuleiten. Bereits Leadermann (1943) und Rabotnow (1948) haben vorgeschlagen, den Faktor L in Glg. (2) als zeitunabhängigen Verschiebungsfaktor in $\sigma$ - oder $\varepsilon$ - Richtung der Isochronen zu definieren, der nur von der jeweiligen Spannung oder Dehnung abhängt. Wenn weiterhin vorausgesetzt wird, daß sich L nur wenig bei Kriech- und Relaxationsbelastung unterscheidet $\mathrm{L}_{\mathrm{c}} \approx \mathrm{L}_{\mathrm{r}}=\mathrm{L}$, kann die elastische Näherung auch im nichtlinear-viskoelastischen Bereich mit brauchbarer Genauigkeit angewendet werden. Experimentelle Erfahrung belegt diese Aussage.

Die Benutzung der Glg. (2) erfordert zunächst die Kenntnis der Kennwerte $\mathrm{E}_{0(\mathrm{t} t} ; \varepsilon_{\mathrm{L}(\mathrm{t})}$ oder $\sigma_{\mathrm{L}(\mathrm{t})}$, die aus entsprechenden Datensammlungen bei der jeweils zutreffenden Zeit und Temperatur verfuigbar sind. Bei der Festlegung der Linearitätsgrenze $\left(\varepsilon_{\mathrm{L}} ; \sigma_{\mathrm{L}}\right)$ ist übergroße Genauigkeit nicht sinnvoll. Es genügt, wenn man sich auf Linearitätsabweichungen unter $5 \%$ festlegt. Bezüglich der $\mathrm{E}_{0(\mathrm{t})^{-}}$ Werte sind Abweichungen von ca. $\pm 10 \%$ selbst für genauere Berechnungen noch ausreichend, da die empfohlenen Sicherheitsbeiwerte solche Streuungen ausreichend abdecken. Hinzu kommen ohnehin unvermeidbare Eigenschaftsschwankungen durch Verarbeitungsprozesse und andere Imponderabilien. Für die Entwurfsphase sind grobe Abschätzungen häufig ausreichend. 
Gegenstand des Vortrages ist die Formulierung einer Methode zur Bestimmung von $\mathrm{L}=f(\sigma ; \varepsilon)$. Die Lösung erfordert eine mathematische Approximation der $\sigma$ - $\varepsilon$-Kurven durch ein geeignetes Stoffgesetz. Eine diesbezuigliche Recherche der Fachliteratur über mehrere Jahrzehnte fördert solche „Gesetze“ im Dutzend zutage. Dazu gehören Potenzpolynome, aus denen bei variabler Anzahl der Glieder beliebig genaue Regressionsansätze (z. B. für die Computerverarbeitung) formulierbar sind, weiterhin Sinushyperbolicus- und Exponentialansätze, denen manchmal eine physikalische Basis (z. B. aus der Platzwechseltheorie) zugeschrieben wird. Einige dieser Ansätze approximieren zwar sehr gut die ISD, sind aber nicht oder nur in sehr unanschaulicher Form zur Berechnung eines zeitunabhängigen Linearitätsfaktors geeignet $/$ z. B. $2 ; 3 /$.

Der Verfasser hat bereits zur TECHNOMER '75 eine Methode zur Bestimmung von $\mathrm{L}=f(\sigma ; \varepsilon)$ vorgeschlagen /4/, deren modifizierte Form /5/ durch neuere Untersuchungen $/ 6$ / sehr gut bestätigt wurde. Ausgangspunkt dieser Methode ist der empirische Tatbestand, daß sich der Tangentenmodul als Funktion der Spannung hinreichend genau nach dem Schema in Bild 3 verhält.

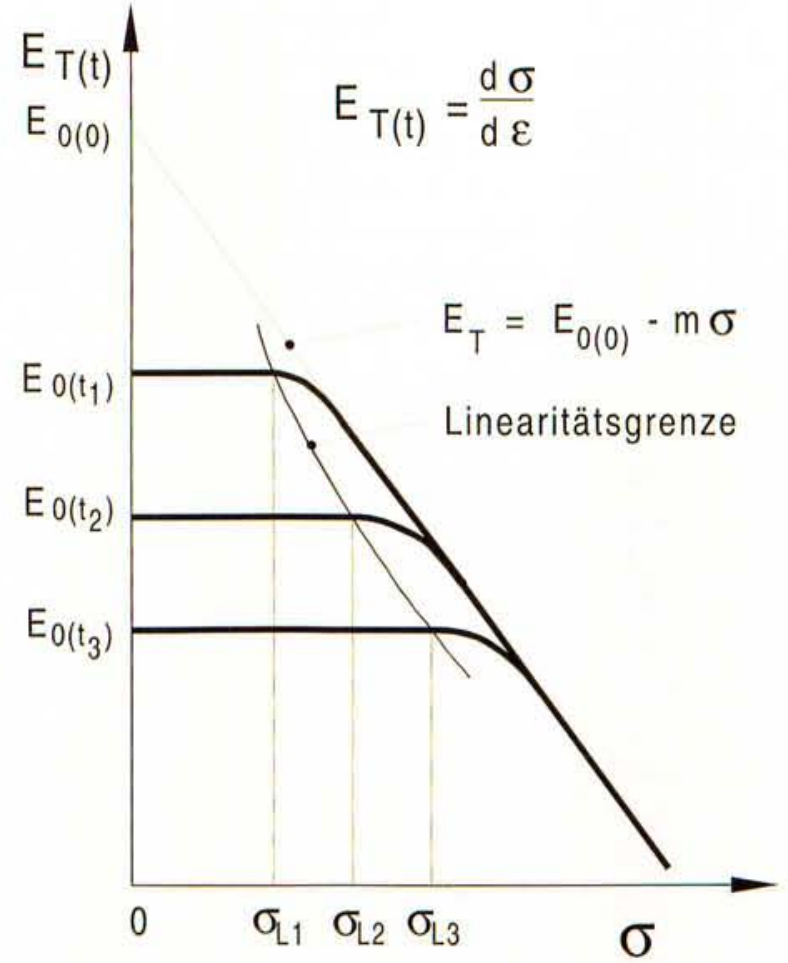

Bild 3: Tangentenmodul als Funktion der Spannung

Aus Bild 3 läßt sich folgende Differentialgleichung ableiten:

$$
E_{T(t)}=\frac{d \sigma}{d \varepsilon}=E_{0(t)}-m \cdot\left(\sigma-\sigma_{L(t)}\right)
$$

Die Besonderheit von Glg. (3) besteht vor allem darin, daß der Anstiegsfaktor $m$ als nahezu zeitunabhängig anzusehen ist, wie auch die Versuchsergebnisse von Lustig /6/ bestätigt haben. Glg. (3) gilt fuir Thermo- und Duroplaste einschließlich Polymerblends sowie fuir gefuillte und verstärkte Kunststoffe, soweit sie ausreichend hartelastisch $\left(\mathrm{E}_{0(\mathrm{t})}>300 \mathrm{~N} / \mathrm{mm}^{2}\right)$ sind. Sie gilt nicht für gummielastische oder weichelastische Polymere. Im Übergangsbereich zwischen Linearitätsgrenze und Einmündung in die abfallende Gerade liegt eine gewisse Unbestimmtheit durch veränderliche $m$-Werte vor. Da dieser Bereich meist sehr klein ist, kann ohne wesentliche Genauigkeitseinschränkung mit dem m-Wert der Hauptgeraden gerechnet werden. Man könnte aber auch den Schnittpunkt der $\mathrm{E}_{O(t)}$-Parallele mit der Hauptgeraden als Linearitätsgrenze definieren. Die Integration von Glg. (3) ergibt folgende Ausdrüicke für $\sigma, \varepsilon$ und $\mathrm{L}$ :

$$
\begin{aligned}
& \sigma=E_{0(t)}\left\{\varepsilon_{L(t)}+\frac{1-\exp \left[-m\left(\varepsilon-\varepsilon_{L(t)}\right)\right]}{m}\right\}(4) \\
& \varepsilon=\frac{\sigma_{L(t)}}{E_{0(t)}}+\frac{1}{m} \ln \left[\frac{E_{0(t)}}{E_{0(t)}-m\left(\sigma-\sigma_{L(t)}\right)}\right] \\
& L=f(\varepsilon)=\frac{\varepsilon_{L(t)}}{\varepsilon}+\frac{1-\exp \left[-m\left(\varepsilon-\varepsilon_{L(t)}\right)\right]}{(5) \varepsilon} \\
& L=f(\sigma)=\frac{\sigma_{L(t)}+\frac{E_{0(t)}}{m} \ln \left[\frac{\sigma}{\left.E_{0(t)}-m_{\left(\sigma-\sigma_{L(t)}\right.}\right)}\right]}{E_{0(t)}}
\end{aligned}
$$

Die Auswertung der Glg. (4) und (5) kann durch spannungs- und dehnungsinvariante Diagramme erleichtert werden. Auf derartige Darstellungen wird hier nicht eingegangen, da schon programmierbare Taschenrechner den Rechenaufwand minimieren. In Tabelle 1 ist auszugsweise eine Kennwertmatrix als Beispiel dargestellt /7/.

\begin{tabular}{|l|c|c|c|c|}
\hline \multirow{2}{*}{$t h$} & \multicolumn{2}{|c|}{$T=23^{\circ} \mathrm{C}$} & \multicolumn{2}{c|}{$\mathrm{T}=80^{\circ} \mathrm{C}$} \\
\cline { 2 - 5 } & $E_{O(t)} \mathrm{N} / \mathrm{mm}^{2}$ & $\mathrm{e}_{\mathrm{L}(\mathrm{t})} \%$ & $\mathrm{E}_{0(\mathrm{t})} \mathrm{N} / \mathrm{mm}^{2}$ & $\mathrm{e}_{\mathrm{L}(\mathrm{t})} \%$ \\
\hline 0 & 2500 & - & 1900 & - \\
\hline 0,1 & 2300 & 0,30 & 1700 & 0,30 \\
\hline 1 & 2200 & 0,35 & 1600 & 0,35 \\
\hline $10^{1}$ & 2100 & 0,38 & 1450 & 0,40 \\
\hline $10^{2}$ & 1950 & 0,40 & 1300 & 0,45 \\
\hline $10^{3}$ & 1800 & 0,45 & 1200 & 0,50 \\
\hline $10^{4}$ & 1700 & 0,55 & 1100 & 0,65 \\
\hline$m$ & \multicolumn{2}{|c|}{50} & \multicolumn{2}{|c|}{45} \\
\hline
\end{tabular}

Tabelle 1: Deformationskennwerte für Polycarbonat (PC)

Auf die Möglichkeiten, die Kennwerte $E_{0(t)}$ und $\varepsilon_{L(t)}$ durch mathematische Approxiationsbeziehungen als Funktionen von Zeit und Temperatur zu beschreiben, soll hier nicht eingegangen werden.

Beispiele für $\mathrm{E}_{0}=f(\mathrm{t})$ mittels Findley-Ansatz finden sich in $/ 1 /$ und $/ 2 /$.

Zusammenfassend lassen sich die Vorteile der Glg. (5) in Verbindung mit Glg. (2) wie folgt bewerten: 
- Der zeitunabhängige m-Wert ermöglicht eine Reduzierung des Prüfaufwandes bei Zeitstandversuchen. Bei einem Prüfzeithorizont von ca. $1000 \mathrm{~h}$ und wenigen Spannungswerten kann mit relativ großer Sicherheit über zwei Zeitgrößenordnungen extrapoliert werden.

- Die Temperaturabhängigkeit der $\mathrm{m}$-Werte ist relativ gering, sofern die Temperaturen hinreichenden Abstand zu molekularen Haupt- oder Nebenrelaxationsgebieten (Erweichungsbereichen) haben.

- Die Glg. (5) ermöglicht eine relativ genaue Anwendung der elastischen Näherung nach Glg. (2) auch für den nichtlinearen Deformationsbereich der Kunststoffe.

- Alle erforderlichen Deformationskennwerte können in einfacher und übersichtlicher Weise (z. B. über Datenbanken) dem Nutzer zur Verfügung gestellt werden. Sie sind problemlos in Computersoftware zu integrieren.

- Die vorstehend angegebene Methode läßt sich nach bisheriger Erkenntnis für alle Konstruktionskunststoffe anwenden.

- Abweichungen der m-Werte von $\pm 20 \%$ sind mit den meisten Berechnungen noch verträglich. Es scheint nach bisherigen Erfahrungen möglich, ganzen Kunststoffgruppen einheitliche Werte zuzuordnen.

\subsection{Kombinierte Beanspruchung}

Werden Bauteilquerschnitte gleichzeitig durch Normalund Schubspannungen beansprucht bzw. liegt ein mehrachsiger Beanspruchungszustand vor, kann Glg. (2) in folgender Modifizierung genutzt werden.

$$
\sigma_{\mathrm{V}}=\mathrm{E}_{0(\mathrm{t})} \cdot \mathrm{L} \cdot \varepsilon_{\mathrm{V}}
$$

In Glg. (6) ist $\sigma_{\mathrm{V}}$ die Vergleichsspannung und $\varepsilon_{\mathrm{V}}$ die Vergleichsdehnung nach der Gestaltänderungsenergiehypothese, die in bekannter Weise zu berechnen sind $/ 1 /$. Zur Berechnung mehrachsiger Spannungs- oder Verformungszustände wird die Querkontraktionszahl $v_{(t)}$ als zeit- und temperaturabhängiger Kennwert benötigt. Ist ein Zahlenwert $v_{0}$ für die Bezugszeit $t_{0}$ und Bezugstemperatur $\mathrm{T}_{0}$ im linearviskoelastischen Bereich bekannt, so kann ein beliebiger Wert berechnet werden /1/:

$$
v_{(t)}=0,5-\left(0,5-v_{0}\right) \cdot \frac{E_{0(t ; T)}}{E_{0\left(t_{0} ; T_{0}\right)}}(7)
$$

Glg. (7) setzt voraus, daß bei Langzeitbelastung nur reinelastische Volumenänderung ohne Kriechanteil eintritt. Für isotrope Kunststoffe ist die Voraussetzung experimentell hinreichend bestätigt. Bei Kenntnis von $v_{(t)}$ kann auch die Umrechnung des E-Moduls in den Schubmodul in bekannter Weise vorgenommen werden:

$$
G_{0(t)}=\frac{E_{0(t)}}{2\left(1+v_{(t)}\right)}
$$

Für die Stabilitätsrechnung ist zu beachten, daß der für die Elastizitätstheorie definierte Knick-, Kipp- oder Beulmodul $\mathrm{E}_{\mathrm{K}}$ von Beanspruchungsart und Bauteilgeometrie abhängt, wobei allgemein die Relation $E_{T} \leq E_{K} \leq E_{S}$ gilt. Der Verfasser hat bereits in $/ 5 /$ vorgeschlagen, aus Sicherheitsgründen für Kunststoffe immer den Tangentenmodul $\mathrm{E}_{\mathrm{K}}=\mathrm{E}_{\mathrm{T}}$ zu verwenden.

Mit Glg. (3) ist dieser problemlos aus der Spannung und mit $E_{T(t)}=E_{0(t)} \cdot e^{-m(\varepsilon-E L(t))}$ aus der Stauchung bere- chenbar. Gelegentlich in der Fachliteratur auftretende Modulunterschiede bei Druck- und Zugbelastung $\left(E_{d}>E_{z}\right)$ sind bei isotropen Werkstoffen in der Regel Auswirkungen der Prüfverfahren (z. B. Verformungsbehinderung durch Druckkörperreibung). Die lineare Viskoelastizitätstheorie schließt aus prinzipiellen Grüden Unterschiede bei isotropen Körpern aus.

Die Instabilität eines Bauteils infolge Druck- und/oder Schubbeanspruchung ist so definiert, daß bei zügig steigender Belastung ein kritischer Verformungszustand erreicht wird, nach dessen Überschreitung plötzlich Knikken, Beulen oder Kippen eintritt. Ist bei Langzeitbelastung von Kunststoffen die vorhandene Belastung kleiner als die kurzzeitige Instabilitätslast, so wird infolge unvermeidbarer Verformungen der Bauteile ein Kriechvorgang mit stetig wachsender Bauteilkrümmung ablaufen, der nicht zum vorstehend beschriebenen katastrophalen Versagen führt. Bei diesem Vorgang ändert sich infolge der Verformung die Belastungsgröße (z. B. Biegemoment), so daß streng genommen keine zeitlich konstante Spannung besteht. Infolge der Begrenzung der Krümmung aus Funktionsgründen und der Größe uiblicher Sicherheitsfaktoren kann dieser Vorgang meist hinreichend als quasistatisch mit den üblichen Gleichungen der Stabilitätstheorie berechnet werden, obwohl ein Stabilitätsversagen im konventionellen Sinne nicht eintritt.

Viele praktischen Belastungsfälle lassen sich auf eine periodisch intermittierende Kriechbelastung nach dem Schema in Bild 4 zurückführen. Vom Verfasser wurde eine Berechnungsmöglichkeit entworfen, deren Parameter hier erklärt sind. Sie geht von einem quasistatischen Ursprungsmodul $\mathrm{E}_{0(\mathrm{~N})}$ nach Rechenvorschrift aus, der wie folgt zur Verformungsberechnung verwendet wird:

$$
\sigma_{\mathrm{V} \max }=\mathrm{E}_{0(\mathrm{~N})} \cdot \mathrm{L} \cdot \varepsilon_{\mathrm{V}}
$$

Glg. (9) stimmt sehr gut mit empirischen Ergebnissen von Brüller/Brand /8/ überein und folgt weitgehend der Konzeption des Binder-Verfahrens /9/.

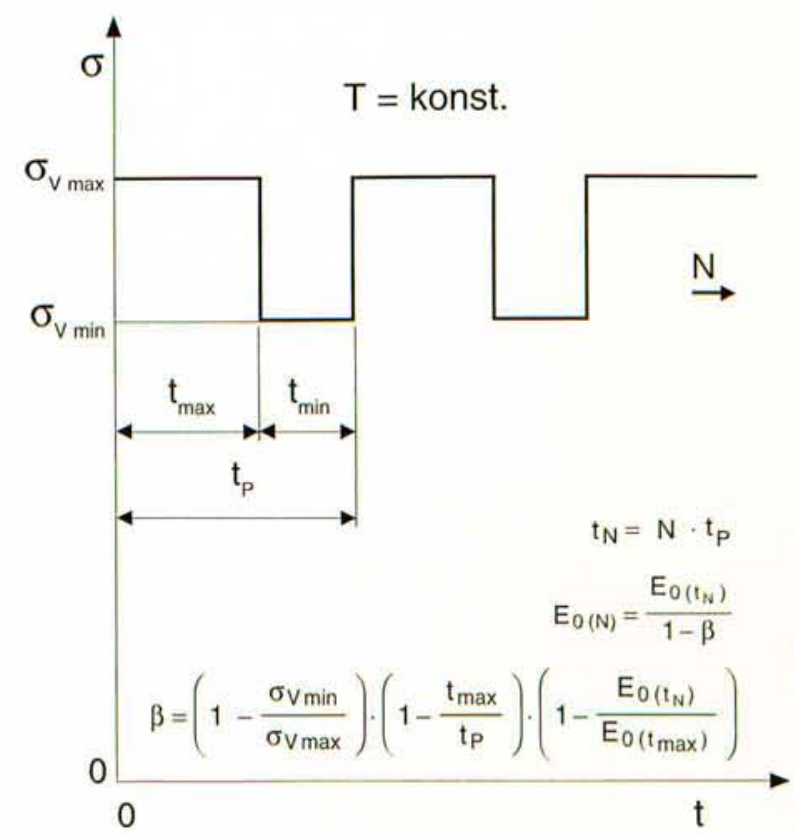

Bild 4: Parameter der periodisch intermittierenden Kriechbelastung (Rechteckverlauf) 
Alle im Vortrag behandelten Verformungsberechnungen setzen zunächst isotherme Belastung voraus. Generell können die Näherungsverfahren / 9 / für wechselnde Temperaturen (nichtisotherme Belastung) auch darauf angewendet werden. Wie im Fall ungleichmäßiger Spannungsverteilung im Querschnitt (z. B. Biegung) der Linearitätsfaktor korrigiert werden kann, ist ansatzweise in /5/ dargestellt. Eine genauere Erläuterung übersteigt den Rahmen dieses Vortrages.

\section{Literatur}

/1/ Göldner, H.; Bergander, H. u.a.: Lehrbuch Höhere Festigkeitslehre Bd.2; Fachbuchverlag Leipzig 1989

12/ Reichelt, E.: Langzeit-Deformationsverhalten von Kunststoffen; Kunststoffe 76(1986)10, S. 971-974

/3/ Sarabi, B.: Ermittlung von Langzeitbemessungskennwerten für Kunststoffe; Kunststoffe 76(1986)4, S. 365-368

14/ Meyer, B.-R.: Invariantes Deformationsgesetz zur Beschreibung des Deformationsverhaltens von festen Hochpolymeren; Vortrag zur TECHNOMER '75 Karl-Marx-Stadt

15/ Starke, L.; Knauer, B.; Meyer, B.-R. u.a.: Konstruktiver Plasteinsatz; Verlag für Grundstoffindustrie Leipzig 1977

/6/ Lustig, V.; Hufenbach, W.: Aussagefähigkeit mechanischer Kennwerte für die Qualitätssicherung von beanspruchten Kunststofformteilen; Vortrag TECHNOMER ' 95 Chemnitz

17/ Trampe, M.; Augustin, A.: m-Wertbestimmung für Thermoplaste; Studienarbeit TFH Wildau 1997

18/ Brüller, O. S.; Brand, N.: Voraussage der Dehnung von Kunststoffen bei zyklischer Be- und Entlastung im linearviskoelastischen Bereich; Kunststoffe 67 (1977)9, S. 527-530

/9/ Binder, K.: Langzeitige Kriech- und Erholungsprozesse von Plastomeren bei Anwendung konstanter und intermittierender Zugbeanspruchungen; Diss. TH Wien 1974

\section{Verfasser}

\section{Prof. Dipl.-Ing. Bernd-Rüdiger Meyer}

Technische Fachhochschule Wildau

Fachbereich Ingenieurwesen/Wirtschaftsingenieurwesen

Tel. (0 33 75) 507-140 